\title{
Difficult airway management in a case with lingual tonsil hypertrophy and temporo-mandibular joint partial ankylosis
}

\author{
Min A Kwon, Jaegyok Song, and Keekeun Park \\ Department of Anesthesiology and Pain Medicine, Dankook University College of Medicine, Cheonan, Korea
}

The lingual tonsil, a normal component of Waldeyer's ring, consists of lymphoid tissue located at the base of tongue [1]. A supraepiglottic mass like lingual tonsillar hypertrophy (LTH) is recognized as a risk factor for unanticipated failed tracheal intubation, and lingual tonsil hypertrophy has been reported to be a cause of difficult airway [1]. Mask ventilation may be difficult or impossible in proportion to the degree of hypertrophy. We report a case of difficult airway resulting from LTH combined with moderate temporo-mandibular joint ankylosis. We obtained a written, informed consent from the patient about using the information of the patient.

A 62-year-old male patient (Height $165 \mathrm{~cm}$, weight $67 \mathrm{~kg}$ ) was scheduled for radicular cyst excision in the anterior maxilla. In the physical examination, there was moderately limited neck mobility, $4 \mathrm{~cm}$ mouth opening with moderately retracted mandible, $6 \mathrm{~cm}$ thyromental distance, and Mallampati class III. Preoperative blood tests, electrocardiogram, and chest radiograph were normal. His past medical history was unremarkable except for well controlled hypertension.

Following placement of routine monitoring and preoxygenation, anesthesia was induced with $60 \mathrm{mg}$ lidocaine, 100 mcg fentanyl, $120 \mathrm{mg}$ propofol, in combination with $50 \mathrm{mg}$ rocuronium. Unexpectedly, mask ventilation was difficult, needing an oral airway and two person mask ventilation. When we gave positive pressure to the airway of the patient, we could see his cheek and submandibular area bulging like a balloon as if there was an obstruction in the supraglottic area. Laryngoscopy revealed a Grade 4 Cormack and Lehane view and BURP (backward upward right pressure) maneuver could not improve the view. An experienced anesthesiologist performed oral intu- bation with flexible fiberoptic bronchoscopy (FOB). However, the tongue base totally obstructed the pharyngeal cavity, and we could not advance the bronchoscope. We tried to clear the airway with chin lift with neck extension, but unexpectedly jaw protraction was almost impossible due to temporo-mandibular (TM) joint partial ankylosis. We tried direct laryngoscopy with a \#3 Macintosh blade to retract the tongue base to make way for FOB. We could advance the fiberoptic bronchoscope under the laryngoscope blade and a normal shaped epiglottis and vocal cords were partially visible. A \#7.5 cuffed plain endotracheal tube was inserted successfully. Following completion of the surgery, the muscle relaxation was recovered, and the patient was extubated without incident.

After surgery, the patient was referred to otolaryngology, and a massive LTH was confirmed with direct laryngoscopy and computed tomography (Fig. 1). His subsequent course was uneventful, and he was discharged home 3 days later.

The etiology of LTH is not known precisely. Mainly, hypertrophy may occur due to compensation for the removal of palatine tonsils, chronic infections, allergy, and reflux of gastric contents [1]. LTH can occupy the entire vallecula and override the tip and lateral borders of the epiglottis. LTH can displace the epiglottis posteriorly, causing dysphagia and respiratory obstruction. Most LTH is asymptomatic and is not visible during the regular physical examination [1].

Ovassapian et al. [1] analyzed 33 patients who had a history of failed endotracheal intubation and difficult airway. The only common finding was LTH. Sixty-four percent of the patients' airway risk index scores were estimated at 0 to 1 which means 95\% chance of easy intubation. There were 12 patients with dif-

Corresponding author: Jaegyok Song, M.D., Ph.D., Department of Anesthesiology and Pain Medicine, Dankook University College of Medicine, 16-5, Anseo-dong, Cheonan 330-715, Korea. Tel: 82-41-550-6819, Fax: 82-41-550-6819, E-mail: drjack@nate.com

(c) This is an open-access article distributed under the terms of the Creative Commons Attribution Non-Commercial License (http:// creativecommons.org/licenses/by-nc/3.0/), which permits unrestricted non-commercial use, distribution, and reproduction in any medium, provided the original work is properly cited. 

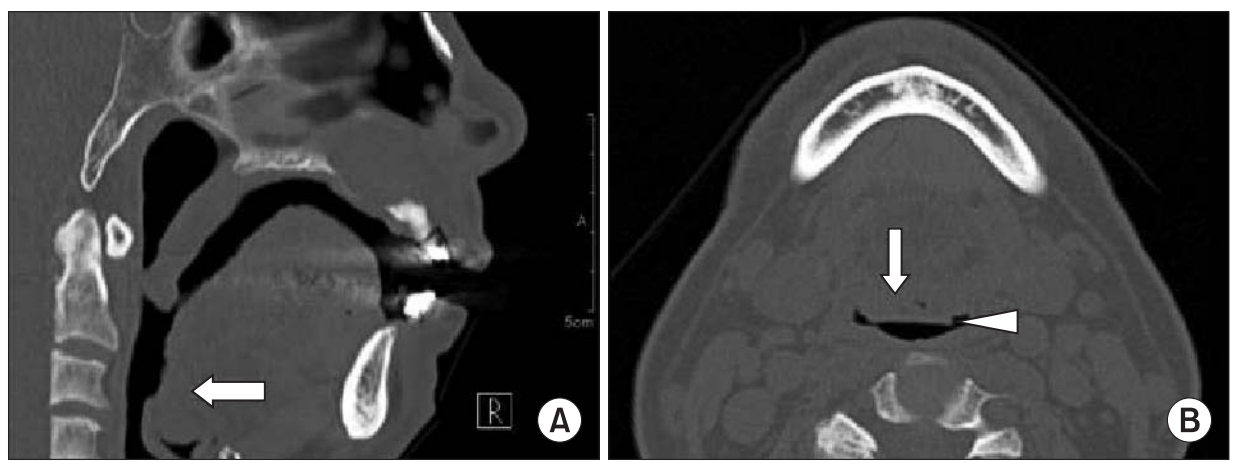

Fig. 1. (A) Computed tomography of head and neck; sagittal view, (B) axial view: the arrow points to lingual tonsil hypertrophy and the arrow head indicates the epiglottis.

ficult mask ventilation.

Various methods are proposed to manage difficult airway due to LTH. A lateral neck x-ray provides useful documentation regarding tongue position and relative size of lingual tonsils [2]. Neuromuscular blocking agents should be avoided during the induction of general anesthesia to preserve the skeletal muscle support of the upper airway [3]. The Miller straight blade laryngoscope is preferred as it is designed so that its tip passes posterior to the epiglottis to elevate it directly, providing better visualization of the glottis opening [4]. However, the Macintosh blade may carry the risk of traumatic injury to the lingual tonsils, with bleeding in the upper airway. Ovassapian et al. [1] reported that if ventilation via face mask or laryngeal mask airway is acceptable and oxygenation is well maintained, fiberoptic intubation can be applied successfully. FOB can evaluate the airway safely and atraumatic intubation is possible.

In this case, there was the limited neck extension with TM joint partial ankylosis due to degenerative change. Head extension and mandible protrusion are crucial factors in increasing the view of the glottis inlet from the FOB under general anesthesia and muscle relaxation [5]. Thus, we performed direct laryngoscopy to retract lingual tonsil and make a passage for FOB. It would be much easier if we tried awake intubation, but we could not expect LTH which can block the insertion of FOB.

Multiple intubation attempts with various methods under general anesthesia can aggravate the patient's airway condition and can be life threatening [5]. Video laryngoscope can be another noninvasive solution of LTH. In our case, video laryngoscope could retract tongue base and show the view of glottis by itself. In this regard, various conditions like unexpected difficult airway after the induction of general anesthesia, difficult mask ventilation, shortage of time, and not experienced with FOB, video laryngoscopy would be better choice than FOB.

The use of laryngeal mask airway (LMA) is controversial. LMA can be a life-saving tool in cannot intubate, cannot ventilate situation, but multiple LMA insertion may induce airway trauma, bleeding, and edema. If non-invasive airway management is failed we should consider invasive airway. Cricothyroidotomy, wire guided retrograde intubation can be considered [5].

In conclusion, we experienced difficult airway management due to LTH with TM joint ankylosis successfully intubated with FOB. LTH cannot be detected with routine physical examination and may induce cannot ventilate and cannot intubate situation. Awake intubation or preservation of self-respiration during airway management is preferred. Fiberoptic bronchoscopy is a gold standard method of non-invasive airway management and video laryngoscopy can be an alternative solution. Sometimes, invasive airway management technique such as wire guided retrograde intubation, cricothyroidotomy and tracheostomy may be selected. Anesthesiologists should be familiar with these techniques.

\section{References}

1. Ovassapian A, Glassenberg R, Randel GI, Klock A, Mesnick PS, Klafta JM. The unexpected difficult airway and lingual tonsil hyperplasia: a case series and a review of the literature. Anesthesiology 2002; 97: 124-32.

2. Cheng KI, Yun MK, Chang MC, Lee KW, Huang SC, Tang CS, et al. Fiberoptic bronchoscopic view change of laryngopharyngeal tissues by different airway supporting techniques: comparison of patients with and without open mouth limitation. J Clin Anesth 2008; 20 : 573-9.

3. Bower CM. Lingual tonsillectomy. Oper Tech Otolaryngol Head Neck Surg 2005; 16: 238-41.

4. Al Shamaa M, Jefferson P, Ball DR. Lingual tonsillar hypertrophy: airway management using straight blade direct laryngoscopy. Anesth Analg 2004; 98: 874.

5. Schroeder K, Becker A, Guite C, Arndt G. Severe lingual tonsillar hypertrophy and the rationale supporting early use of wire-guided retrograde intubation. Saudi J Anaesth 2010; 4: 102-4. 